\title{
Novel and unique domains in aminoacyl-tRNA synthetases from human fungal pathogens Aspergillus niger, Candida albicans and Cryptococcus neoformans
}

\author{
Manish Datt and Amit Sharma*
}

\begin{abstract}
Background: Some species of fungi can cause serious human diseases, particularly to immuno-compromised individuals. Opportunistic fungal infections are a leading cause of mortality, and present an emerging challenge that requires development of new and effective therapeutics. Aminoacyl-tRNA synthetases (aaRSs) are indispensable components of cellular protein translation machinery and can be targeted for discovery of novel anti-fungal agents.

Results: Validation of aaRSs as potential drug targets in pathogenic microbes prompted us to investigate the genomic distribution of aaRSs within three fungi that infect humans - A. niger, C. albicans and C. neoformans. Hidden Markov Models were built for aaRSs and related proteins to search for homologues in these fungal genomes. Here, we provide a detailed and comprehensive annotation for 3 fungal genome aaRSs and their associated proteins. We delineate predicted localizations, subdomain architectures and prevalence of unusual motifs within these aaRSs. Several fungal aaRSs have unique domain appendages of unknown function e.g. A. niger AsxRS and C. neoformans TyrRS have additional domains that are absent from human homologs.

Conclusions: Detailed comparisons of fungal aaRSs with human homologs suggest key differences that could be exploited for specific drug targeting. Our cataloging and structural analyses provide a comprehensive foundation for experimentally dissecting fungal aaRSs that may enable development of new anti-fungal agents.
\end{abstract}

Keywords: Aminoacyl-tRNA synthetases, Domain organization, Drug targets, Fungal pathogens

\section{Background}

Fungal infections are emerging as a leading cause of human mortality in hospital settings [1-5]. Recent trends suggest that select species of fungi have the ability to take advantage of debilitated immune system to cause mortality [1-5]. Data suggest that diseases caused by opportunistic fungi result in scores of human deaths worldwide, and hence containment of human fungal infections is a pressing challenge [1-5]. The pathogenesis of such opportunistic fungi is a complex phenomenon since these organisms more often than not lack dedicated 'virulence' factors [1-5]. Therefore, understanding cellular and molecular

\footnotetext{
*Correspondence: amit.icgeb@gmail.com

Structural and Computational Biology group, International Center for Genetic Engineering and Biotechnology (ICGEB), New Delhi 110067, India
}

attributes of these fungal pathogens is indispensible towards developing effective therapeutics.

Aspergillus, Candida, and Crytococcus are the three most common genus of fungi associated with infectious diseases in humans [1-5]. Candidiasis or thrush is the most prevalent fungal infection in humans, commonly caused by C. albicans. Candida is generally present on the skin and mucous membrane and does not cause infection, however invasive candidiasis occurs when Candida enters the bloodstream and spreads throughout the body [6]. Immuno-compromised (as in case of cancer and AIDS patients) individuals are particularly prone to C. albicans infections [7]. Aspergillus species causes second most common fungal infections in humans [8]. A. fumigates is the most virulent species in this genus, and cases of pulmonary aspergillosis have been reported resulting from A. niger 
infections as well [9]. Cryptococcosis is a fungal disease caused primarily by the two species Cryptococcus neoformans and Cryptococcus gattii [10]. C. neoformans is present in soil and spreads through microscopic airborne fungal spores [11]. C. neoformans infection increases the probability of re-hospitalization of AIDS patients who otherwise show positive response to anti-retroviral therapy [7,12]. $A$. niger and $C$. albicans belong to Ascomycota phylum while C. neoformans belongs to Basidomycota phylum within the fungal kingdom. Genome sizes for A. niger, C. albicans, and $C$. neoformans are $\sim 33 \mathrm{Mb}$ (19 chromosomes), $\sim 14$ $\mathrm{Mb}$ (8 chromosomes), and $\sim 19 \mathrm{Mb}$ (14 chromosomes). Predicted number of ORFs (open reading frames) in $A$. niger, C. albicans, and C. neoformans are $14000, \sim 6200$, and $\sim 6500$. In addition to genome sequencing, transcriptomics analyses for these three fungi are also available [13-15].

Aminoacyl-tRNA synthetases (aaRSs) drive protein translational machinery by catalyzing the addition of amino acids to cognate tRNA $[16,17]$. Structural studies have confirmed their modular architecture, with separate domains for aminoacylation and tRNA binding [16-18]. Based on their structural fold and mode of tRNA binding these enzymes have been classified into class I and II aaRSs [16-18]. In addition to performing translational activities, these proteins also localize within mitochondria and apicoplasts to participate in synthesis of proteins encoded by genomes within organelles [16-21]. Aside from their translational functions, aaRSs are implicated in various noncanonical functions such as gene transcription, mRNA translation, inflammation, and immune response [16-21]. Therefore, aaRSs constitute a very important subset of proteins in any genome, and inhibition of their enzymatic activity can be deleterious for the host. Ever since the potential of malaria parasitic aaRSs for exploration as new drug targets has been highlighted [19,22], intense efforts from many groups have yielded an array of druggable malaria parasite aaRSs [23-35]. More recently, protein translation components from other eukaryotic pathogens like Leishmania [36] and Toxoplasma [21] have also been explored. Hence, experimental dissection of critical translation components like aaRSs is on high priority as one avenue of novel target discovery in pathogen biology. Dearth of structural characterization of larger number of fungal aaRSs severely restricts scope for targeted development of new anti-aaRS drugs, despite the acceptance of aaRSs as druggable targets. In context of this work, $C$. albicans SerRS is the only full-length aaRS from these three fungal genomes for which crystal structure has been solved [37]. Here, we have studied aaRSs from $A$. niger, $C$. albicans and $C$. neoformans using profile-based Hidden Markov Models (HMM). In addition, homologs for aaRSrelated proteins such as the editing domains (AlaX) and Dtyrosyl-deacylase (DTD), Gln and Asn amidotransferases, and aaRS-associated $\mathrm{P} 43$ protein have also been identified. Protein sequences of thus identified aaRSs have been comprehensively annotated in context of their domain organization. We discovered novel domain appendages in several fungal aaRSs that are absent from human homologs. Our results highlight key structural attributes of fungal aaRSs that could be exploited for drug targeting to combat mycoses in humans.

\section{Methods}

Retrieval of fungal genome sequences and aaRS dataset Computationally translated open reading frames (ORFs) for $A$. niger and $C$. albicans were downloaded from their corresponding genome databases available at http://www. aspergillusgenome.org/ and http://www.candidagenome. org/ respectively. Similarly, ORF sequences for $C$. neoformans were retrieved from FungiDB [38]. To annotate aaRSs and aaRS-associated proteins, a dataset of annotated aaRSs and associated proteins was prepared from the UniProt database [39]. Our dataset included protein sequences of human aaRSs, human aaRS trans-editing domain (DTD, AlaX), Gln and Asn amidotransferases, aaRS associated protein P43 and Ybak protein from E. coli. The UniProt accession numbers for sequences in the dataset are given in supplementary Additional file 1: Table S1.

\section{Generation of Hidden Markov Models (HMMs), protein annotation and modeling}

All protein sequences from our dataset were individually used to perform BLAST searches against non-redundant databases available at NCBI. Top 1000 homologs from each BLAST search were saved for further analyses as we reasoned that these may effectively capture the evolutionary diversity at different positions within aaRSs, thus facilitating generation of robust HMMs. For each aaRS, HMMs were generated using the homologs identified from BLAST search. Sequence Alignment and Modeling Software System (SAM) [40] was used to generate MSA, and HMMER package was used for building profile HMM for each MSA. Significantly similar matches for each HMM profile were identified within the genomic sequences from the three fungi. Pfam [41] domains were assigned to predicted aaRS sequences from fungal genomes. Sub-cellular localization for sequences showing significant HMM search score was annotated using Wolf PSORTb webserver using default parameters optimized for fungal proteins [42]. For a query protein sequence, this server gives a relative score to each subcellular location and the highest scoring compartment has been used here to annotate aaRSs. MitoProt [43] and TagetP [44] servers were used to identify signal sequences within the predicted mitochondrial aaRSs. Sequence alignment of aaRSs was performed using Tcoffee program with default parameters [45]. Structural 
conservation was mapped onto the sequence alignment using Expresso options available at http://tcoffee.crg.cat/. Homology modeling for protein sequences was performed using Phyre2 server [46] and PyMol [47] was used for structure visualization and analyses.

\section{Results and discussion}

We have employed a profile HMM-based search to computationally identify aaRSs in the three fungal genomes. Our results show that there are a total of 29 aaRSs in $A$. niger, 28 in C. albicans and 26 in C. neoformans (Tables 1 , 2 and 3). This exercise enabled us to annotate aaRSs specific for all 20 amino acids in these fungi (Tables 1, 2 and 3). All predicted aaRSs had highly significant e-values based on profile HMM searches. Functional annotation of these aaRSs was cross-validated using BLAST searches against conserved domain database (CDD) at NCBI. Amongst the three fungi, maximum variation in the full length of aaRSs was observed in $A$. niger genome with the largest and smallest proteins being IleRS (212883-mRNA) with 1524 residues and TrpRS (209919-mRNA) with 391 residues respectively (Table 1 ). Interestingly, for some aaRSs there exist multiple enzymes for charging tRNAs that are specific to a particular amino acid (Figure 1). Our results show that there are $8 \mathrm{~A}$. niger aaRSs that are present in two versions (AspRS, GluRS, GlyRS, IleRS, LeuRS, PheRS, TrpRS and AsxRS), 7 C. albicans aaRSs that are present in two versions (GluRS, IleRS, LeuRS, PheRS, TrpRS, TyrRS and AsxRS), and 5 C. neoformans aaRSs that are present in two versions (AsnRS, GluRS, IleRS, PheRS and TyrRS, Figure 1A). These two versions of aaRSs would presumably facilitate localization in different sub-cellular compartments e.g. in cytoplasm and mitochondria. Comparisons amongst the three fungi show GluRS, IleRS, LeuRS and PheRS to be the common set of two-copy enzymes. To analyze compartmentalization of aaRSs, subcellular localization was calculated for all predicted aaRSs. Expectedly, this showed that whenever two same amino acid aaRSs were present, one was predicted to be cytoplasmic while the other mitochondrial (Tables 1, 2 and 3). We then were able to compile predicted sub-cellular distribution for all aaRSs (Figure 1B), along with putative signal sequences for mitochondrial aaRSs (Additional file 1: Figure S1). Out of total 29 A. niger aaRSs $-16,11$, and 2 aaRS(s) were predicted to (co-) localize in cytoplasm, mitochondria and nucleus (Figure $1 \mathrm{~B}$ and Table 1). In $C$. albicans 13, 9 and 6 aaRSs were predicted to be cytoplasmic, mitochondrial and nuclear (Figure 1B and Table 2). Finally, C. neoformans aaRSs were predicted to be equally partitioned between cytoplasm and mitochondria with 13 aaRSs in each (Figure $1 \mathrm{~B}$ and Table 3). This analysis presents a conundrum where none of the translational compartments in the three fungi seem independent for generating all 20 charged tRNAs. It is likely that some of the identified aaRSs have dual sub-cellular localizations within the cell. In addition, transport of charged tRNAs between cellular compartments could compensate for absence of any particular aaRS in a given chamber like cytoplasm or mitochondria, as in the case of other organisms [48].

We also sought to catalog trans-editing domains within these fungal genomes. D-Tyrosyl-tRNATyr deacylase (DTD) catalyzes hydrolysis of bond between D-aminoacid and tRNA. These enzymes play an important role in evading toxicity resulting from accumulations of D-amino acids within the cell [49]. Trans-editing domains such as AlaX maintain fidelity of tRNA charging by hydrolyzing incorrectly charged amino acid attached to tRNA ${ }^{\text {Ala }}$ [50]. Hence, in addition to profile HMM search for 20 canonical aaRSs; screening for aaRS-associated proteins was also performed (see Methods section for details). Our results suggest that trans-editing domains such as DTD and AlaX are found in all the three fungi (Tables 1, 2 and 3). Similarly, the possibility of alternate route for Gln-tRNA ${ }^{\text {Gln }}$ and Asn-tRNA ${ }^{\text {Asn }}$ synthesis was examined [33,51]. In this alternate mechanism, a non-discriminating aaRS catalyze the synthesis of Glu-tRNA ${ }^{\text {Gln }}$ or Asp-tRNA ${ }^{\text {Asn }}$ followed by catalysis by a corresponding amidotransferase to generate Gln-tRNA ${ }^{\text {Gln }}$ or Asn-tRNA ${ }^{\text {Asn }}$ respectively. Curiously, two separate proteins corresponding to amidotransferase (Asn synthase) were observed in all the three fungal genomes (Tables 1, 2 and 3). The existence of Asn synthase corroborates with the presence of above identified nondiscriminating domains (AsxRS) within the host fungi (Tables 1, 2 and 3). Similar to Asn synthase, Gln synthase is a transamidase that catalyzes the synthesis of GlntRNA $^{\text {Gln }}$ from Glu-tRNA ${ }^{\text {Gln }}$. In mammals, heterotrimeric Gln synthase (GatABC) is frequently observed within mitochondria for synthesis of Gln-tRNA ${ }^{\text {Gln }}$ [52]. In addition to aminoacylation activity, subunit proteins of Gln synthase have been shown to be important for proper functioning of mitochondrial activities unrelated to protein translation [52]. We additionally identified homologs for subunit A and B of Gln synthase in the three fungi (Tables 1, 2 and 3), however, homologs for GatF subunit could be ascertained only in case of $A$. niger and $C$. albicans.

In some eukaryotes, a few aaRSs associate with each other and with auxillary proteins (called P18, P38, and P43) to form multi-synthetase complex (MSC) [53,54]. It has been proposed that MSC improves translational efficiency by channeling tRNAs to aaRSs and ribosomes [55]. In addition, MSC may serve as a reservoir of pro-cytokines and other regulatory molecules that can be released per physiological requirements [56]. A central component of MSC is $\mathrm{P} 43$, a polypeptide of $\sim 43 \mathrm{kDa}$ that harbors tRNA binding domain. P43 is associated with multitude of cellular functions such as protein synthesis, axonal development, 
Table 1 ORFs in A. niger having aaRS or associated domains

\begin{tabular}{|c|c|c|c|c|c|c|}
\hline & aaRS domain & ORF & $\begin{array}{l}\text { Predicted sub-cellular } \\
\text { localization }\end{array}$ & $\begin{array}{l}\text { Protein } \\
\text { length }\end{array}$ & $\begin{array}{l}\text { E-value for HMM } \\
\text { prediction }\end{array}$ & $\begin{array}{l}\text { Identity with human } \\
\text { homolog (\%) }\end{array}$ \\
\hline \multirow[t]{14}{*}{ Class I } & ArgRS & 197261-mRNA & Cyto & 646 & NA & 36 \\
\hline & CysRS & 211236-mRNA & Cyto & 804 & 0 & 39 \\
\hline & GlnRS & 45754-mRNA & Cyto & 625 & $4.4 \mathrm{e}-247$ & 44 \\
\hline & GluRS & 56891-mRNA & Nucl & 712 & 0 & 45 \\
\hline & GluRS & 121468-mRNA & Mito & 427 & $3.5 e-155$ & 37 \\
\hline & lleRS & 52642-mRNA & Cyto & 1077 & 0 & 53 \\
\hline & lleRS & 212883-mRNA & Mito & 1524 & 0 & 36 \\
\hline & LeuRS & 183116-mRNA & Mito & 989 & 0 & 38 \\
\hline & LeuRS & 52554-mRNA & Cyto & 1126 & 0 & 45 \\
\hline & MetRS & 208481-mRNA & Cyto & 659 & 0 & 48 \\
\hline & TrpRS & 54362-mRNA & Cyto & 433 & $6 e-156$ & 55 \\
\hline & TrpRS & 209919-mRNA & Mito & 391 & $6 e-156$ & 40 \\
\hline & TyrRS & 54566-mRNA & Mito & 611 & $5.1 e-123$ & 41 \\
\hline & ValRS & 194077-mRNA & Cyto & 1315 & 0 & 46 \\
\hline \multirow[t]{16}{*}{ Class II } & AlaRS & 51231-mRNA & Mito & 1019 & $7.4 \mathrm{e}-37$ & 48 \\
\hline & AsnRS & 210632-mRNA & Mito & 543 & $1.5 e-146$ & 37 \\
\hline & AspRS & 57039-mRNA & Mito & 676 & $9.5 e-179$ & 38 \\
\hline & AspRS & 56196-mRNA & Cyto & 559 & $3.5 e-281$ & 48 \\
\hline & AsxRS & 207470-mRNA & Cyto_nucl & 574 & $4.7 e-270$ & 50 \\
\hline & AsxRS & 55404-mRNA & Nucl & 952 & 0 & 45 \\
\hline & GlyRS & 57294-mRNA & Mito & 738 & 0 & 51 \\
\hline & GlyRS & 50342-mRNA & Cyto & 667 & 0 & 47 \\
\hline & HisRS & 51854-mRNA & Mito & 619 & $1.4 \mathrm{e}-287$ & 53 \\
\hline & LysRS & 47873-mRNA & Cyto & 604 & 0 & 57 \\
\hline & PheRS & 55517-mRNA & Mito & 514 & $3.8 e-230$ & 38 \\
\hline & PheRS a & 56159-mRNA & Cyto & 518 & $2.8 \mathrm{e}-254$ & 47 \\
\hline & PheRS $\beta$ & 211951-mRNA & Cyto_nucl & 600 & 0 & 47 \\
\hline & ProRS & 210319-mRNA & Cyto & 604 & 0 & 53 \\
\hline & SerRS & 57312-mRNA & Cyto & 474 & $5.2 \mathrm{e}-262$ & 48 \\
\hline & ThrRS & 51819-mRNA & Cyto & 718 & 0 & 50 \\
\hline \multirow[t]{8}{*}{ aaRS related proteins } & AlaX & 189390-mRNA & Cyto & 271 & $1.4 \mathrm{e}-33$ & 24 \\
\hline & DTD & 56197-mRNA & Cyto_mito & 191 & NA & 47 \\
\hline & P43 homolog & 210303-mRNA & Cyto & 445 & $3.1 e-159$ & 42 \\
\hline & Asn synthase & 57091-mRNA & Mito & 573 & $8.8 \mathrm{e}-57$ & 34 \\
\hline & Asn synthase & 186429-mRNA & Cyto & 694 & $1.5 e-39$ & 27 \\
\hline & GatA & 170115-mRNA & Extr & 504 & $1.7 e-159$ & 39 \\
\hline & GatB & 172989-mRNA & Mito & 601 & $2.2 \mathrm{e}-138$ & 33 \\
\hline & GatF & 173432-mRNA & Mito & 886 & $8.1 \mathrm{e}-12$ & NA \\
\hline
\end{tabular}

NA in e-value indicates fungal sequences that could be identified by HMM search and were retrieved from NCBI using keyword search.

glucagon secretion, and autoimmune suppression [57]. Our profile HMM-based screening revealed existence of $\mathrm{P} 43$ variants in all the three fungal genomes (Tables 1, 2 and 3). Presence of MSC and its constituents now remain to be addressed experimentally.
Domain architecture for aaRSs in A. niger

Profile HMM searches identified a total of 29 aaRS in $A$. niger - 14 belonging to class I and 15 to class II. Among class I aaRSs - IleRS, LeuRS, GlnRS, ArgRS, and MetRS had $\mathrm{N}$-terminal catalytic domain followed by anticodon 
Table 2 ORFs in C. albicans having aaRS or associated domains

\begin{tabular}{|c|c|c|c|c|c|c|}
\hline & aaRS domain & ORF & $\begin{array}{l}\text { Predicted sub-cellular } \\
\text { localization }\end{array}$ & Protein length & $\begin{array}{l}\text { E-value for HMM } \\
\text { prediction }\end{array}$ & $\begin{array}{l}\text { Identity with human } \\
\text { homolog (\%) }\end{array}$ \\
\hline \multirow[t]{15}{*}{ Class I } & ArgRS & orf19.3341 & Cyto & 622 & $1.2 \mathrm{e}-104$ & 38 \\
\hline & CysRS & orf19.4931 & Mito & 781 & 0 & 42 \\
\hline & GlnRS & orf19.7064 & Cyto & 799 & 0 & 38 \\
\hline & GluRS & orf19.2415 & Mito & 509 & $1.9 e-199$ & 35 \\
\hline & GluRS & orf19.7057 & Cyto & 725 & NA & 42 \\
\hline & lleRS & orf19.2138 & Nucl & 1088 & 0 & 53 \\
\hline & IleRS & orf19.2382 & Mito & 973 & 0 & 36 \\
\hline & LeuRS & orf19.2560 & Cyto & 1097 & 0 & 46 \\
\hline & LeuRS & orf19.5705 & Cyto & 861 & 0 & 37 \\
\hline & MetRS & orf19.3955 & Cyto & 748 & 0 & 51 \\
\hline & TrpRS & orf19.5226 & Cyto & 424 & $3 e-235$ & 56 \\
\hline & TrpRS & orf19.4299 & $\mathrm{Nucl}$ & 399 & $4.8 \mathrm{e}-156$ & 42 \\
\hline & TyrRS & orf19.2694 & Cyto & 409 & $3.8 e-170$ & 53 \\
\hline & TyrRS & orf19.109 & Mito & 501 & 7.3e-152 & 34 \\
\hline & ValRS & orf19.1295 & Mito & 1119 & 0 & 48 \\
\hline \multirow[t]{14}{*}{ Class II } & AlaRS & orf19.5746 & Cyto & 969 & 0 & 50 \\
\hline & AsnRS & orf19.6698 & Mito & 489 & $6.2 \mathrm{e}-146$ & 35 \\
\hline & AspRS & orf19.4478 & Mito & 698 & $5.5 e-173$ & 35 \\
\hline & AsxRS & orf19.2407 & Cyto & 578 & 0 & 58 \\
\hline & AsxRS & orf19.6702 & Cyto & 552 & $1.3 e-261$ & 52 \\
\hline & GlyRS & orf19.437 & Nucl & 652 & 0 & 50 \\
\hline & HisRS & orf19.4051 & Mito & 501 & $2.3 e-271$ & 52 \\
\hline & LysRS & orf19.6749 & $\mathrm{Nucl}$ & 594 & 0 & 56 \\
\hline & PheRS & orf19.2039 & Mito & 446 & $8.8 e-218$ & 39 \\
\hline & PheRS a & orf19.2960 & Cyto & 496 & $3.2 \mathrm{e}-244$ & 47 \\
\hline & PheRS $\beta$ & orf19.2573 & Cyto_nucl & 592 & $1.1 \mathrm{e}-284$ & 45 \\
\hline & ProRS & orf19.6701 & Cyto & 691 & 0 & 57 \\
\hline & SerRS & orf19.269 & Nucl & 458 & $3.8 e-245$ & 46 \\
\hline & ThrRS & orf19.5685 & Nucl & 706 & 0 & 59 \\
\hline \multirow[t]{8}{*}{ aaRS related proteins } & DTD2 & orf19.297 & Cyto & 163 & NA & 48 \\
\hline & AlaX & orf19.5239 & Cyto_nucl & 471 & $2.1 \mathrm{e}-127$ & 31 \\
\hline & P43 homolog & orf19.2422 & Cyto & 369 & $1.9 e-130$ & 53 \\
\hline & Asn synthase & orf19.3626 & Nucl & 704 & $1 e-178$ & 25 \\
\hline & Asn synthase & orf19.198 & Cyto & 573 & $1.1 \mathrm{e}-58$ & 35 \\
\hline & GatA & orf19.3956 & Mito & 450 & $1.7 e-135$ & 34 \\
\hline & GatB & orf19.2494 & Nucl & 488 & $9.7 e-141$ & 32 \\
\hline & GatF & orf19.4092 & Nucl & 165 & NA & NA \\
\hline
\end{tabular}

NA in e-value indicates fungal sequences that could be identified by HMM search and were retrieved from NCBI using keyword search.

binding domain (ABD) towards the C-terminal (Figure 2). Two different proteins corresponding to IleRS were predicted to localize in cytoplasm (52642-mRNA) and extracellular space (212883-mRNA) respectively. However, the synthetase domain of IleRS (212883-mRNA) shares $~ 36 \%$ sequence identity with human mitochondrial IleRS and therefore its sub-cellular localization is likely to be mitochondrial rather than extracellular. Interestingly, one IleRS (212883-mRNA) contains an addition uncharacterized protein family (UPF0183) domain at its N-terminal (Figure 2). BLAST searches for this IleRS (212883-mRNA) against human genome failed to identify a homolog for the UPF 
Table 3 ORFs in C. neoformans having aaRS or associated domains

\begin{tabular}{|c|c|c|c|c|c|c|}
\hline & aaRS domain & ORF & $\begin{array}{l}\text { Predicted sub-cellular } \\
\text { localization }\end{array}$ & Protein length & $\begin{array}{l}\text { E-value for HMM } \\
\text { prediction }\end{array}$ & $\begin{array}{l}\text { Identity with human } \\
\text { homolog (\%) }\end{array}$ \\
\hline \multirow[t]{13}{*}{ Class I } & ArgRS & CNBG3480 & Cyto & 622 & NA & 37 \\
\hline & CysRS & CNBB4810 & Cyto & 785 & 0 & 42 \\
\hline & GlnRS & CNBA0720 & Cyto & 858 & $5.2 \mathrm{e}-300$ & 40 \\
\hline & GluRS & CNBK2470 & Mito & 588 & $2.3 e-197$ & 35 \\
\hline & GluRS & CNBF4640 & Cyto & 728 & $3.1 \mathrm{e}-295$ & 43 \\
\hline & IleRS & CNBA2140 & Cyto_nucl & 1094 & 0 & 51 \\
\hline & HeRS & CNBN1610 & Mito & 1032 & 0 & 36 \\
\hline & LeuRS & CNBM1150 & Cyto & 1114 & 0 & 46 \\
\hline & MetRS & CNBD2070 & Cyto & 724 & 0 & 46 \\
\hline & TrpRS & CNBJ3070 & Mito & 641 & $5.5 e-221$ & 55 \\
\hline & TyrRS & CNBA1270 & Cyto & 819 & $3.3 e-218$ & 47 \\
\hline & TyrRS & CNBJ0260 & Mito & 478 & $1.2 \mathrm{e}-169$ & 47 \\
\hline & ValRS & CNBB3840 & Mito & 1109 & 0 & 47 \\
\hline \multirow[t]{14}{*}{ Class ॥ } & AlaRS & CNBF2180 & Mito & 1012 & 0 & 49 \\
\hline & AsnRS & CNBD2410 & Mito & 513 & $1.7 e-157$ & 38 \\
\hline & AsnRS & CNBE1330 & Cyto & 600 & $1.8 \mathrm{e}-251$ & 48 \\
\hline & AspRS & CNBK1200 & Mito & 693 & $8.5 e-193$ & 36 \\
\hline & AsxRS & CNBG4380 & Cyto & 884 & 0 & 42 \\
\hline & GlyRS & CNBF3810 & Cyto & 673 & 0 & 46 \\
\hline & HisRS & CNBC0810 & Mito & 586 & $4.2 \mathrm{e}-270$ & 47 \\
\hline & LysRS & $\mathrm{CNBH} 3030$ & Mito & 630 & 0 & 52 \\
\hline & PheRS & CNBC0600 & Mito & 476 & $4.5 e-214$ & 40 \\
\hline & PheRS a & CNBJ1210 & Cyto & 510 & $1.1 e-250$ & 51 \\
\hline & PheRS $\beta$ & CNBC3360 & Nucl & 632 & $4.1 e-283$ & 44 \\
\hline & ProRS & CNBB0150 & Mito & 740 & 0 & 55 \\
\hline & SerRS & CNBD0870 & Cyto & 461 & $2.6 e-225$ & 40 \\
\hline & ThrRS & CNBB5220 & Mito & 772 & 0 & 56 \\
\hline \multirow[t]{7}{*}{ aaRS related proteins } & AlaX & CNBA4150 & Cyto & 523 & $1.1 \mathrm{e}-108$ & 29 \\
\hline & DTD & CNBB3530 & Cyto & 234 & $1 e-41$ & 47 \\
\hline & P43 homolog & CNBF3180 & Cyto & 383 & $1.8 \mathrm{e}-124$ & 47 \\
\hline & Asn synthase & CNBK3010 & Mito & 572 & $1.7 e-196$ & 34 \\
\hline & Asn synthase & CNBL0870 & Mito & 592 & $1.6 \mathrm{e}-65$ & 34 \\
\hline & GatA & CNBD1400 & Mito & 493 & $1.7 e-119$ & 33 \\
\hline & GatB & CNBG4210 & Mito & 521 & $1.7 e-176$ & 37 \\
\hline
\end{tabular}

NA in e-value indicates fungal sequences that could be identified by HMM search and were retrieved from NCBI using keyword search.

part of the sequence (Figure 3A). Two separate proteins for LeuRS were identified, putatively localized to cytoplasm (52554-mRNA) and mitochondria (183116-mRNA). Mitochondrial (121468-mRNA) and potentially nuclear (565891-mRNA) copies of GluRS were also identified. In latter, an additional glutathione-S-transferase C-terminal domain (GST_C, an $\alpha$-helix containing structural domain) is evident at the N-terminus. Human bifunctional GluRS/ ProRS also contains GST_C domain at the N-terminus and these proteins shares $45 \%$ identity with $A$. niger GluRS. The GST_C domain fusion is also present in human MetRS and ValRS. Curiously, the GlnRS (45754mRNA) lacks N-terminal RNA binding region that is present in human and other fungal homologs (Figure 2). The TrpRSs are two-copy enzymes in A. niger, one each for cytoplasm (54362-mRNA) and mitochondria (209919mRNA). In case of CysRS, GluRS, MetRS, TrpRS and TyrRS the Pfam server could only annotate their catalytic 
A

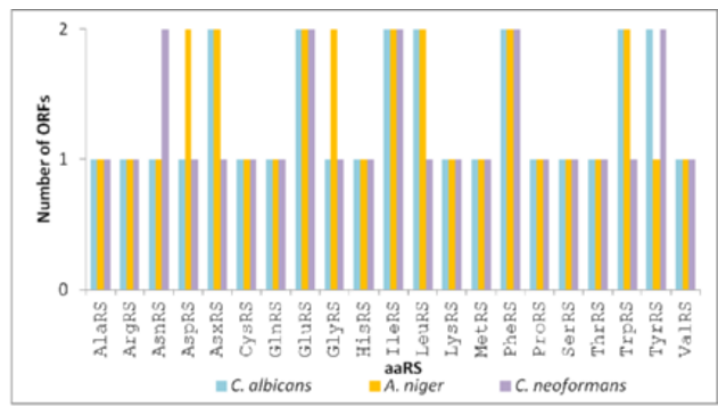

B

—Cytoplasm Mitochondria $\quad$ Nucleus

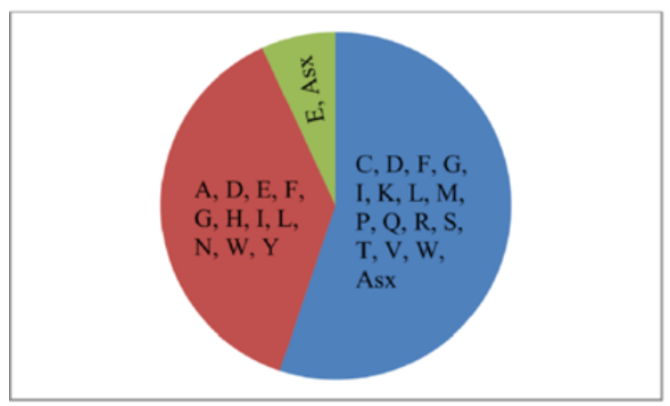

A. niger (29)

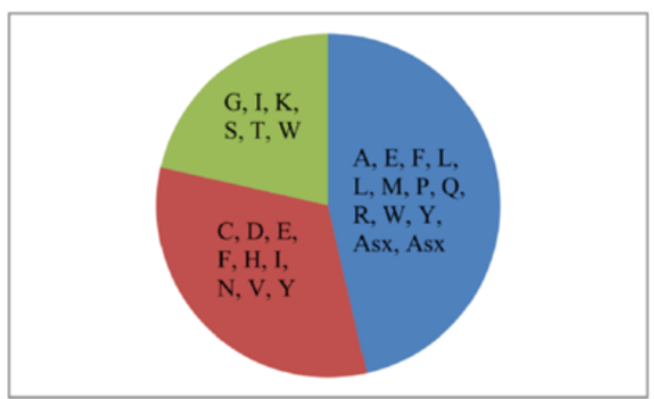

\section{C. albicans (28)}

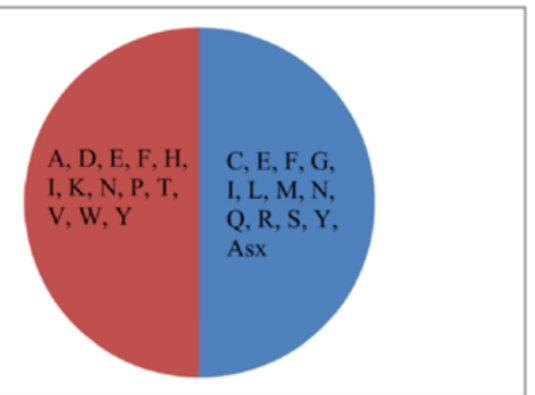

Figure 1 Hidden Markov Model (HMM) based prediction of aaRSs in fungal genomes. (A) Number of predicted aminoacyl-tRNA synthetases (aaRSs) in the three fungal genomes. (B) Predicted distribution of aaRSs in the sub-cellular compartments of the three fungal pathogens. The aaRSs are denoted by the single letter code for their corresponding amino acid and Asx refers to non-discriminating aspartyl-tRNA synthetase.

domains within these sequences. However, based on the catalytic domain positioning it appears likely that their anti-codon binding domains lie towards the C-termini (Figure 2).
Surprisingly, A. niger ValRS (194077-mRNA) displays an additional Brix domain at its C-terminus (Figure 2). Protein families containing Brix domain participate in ribosomal biogenesis and rRNA binding [58]. These 


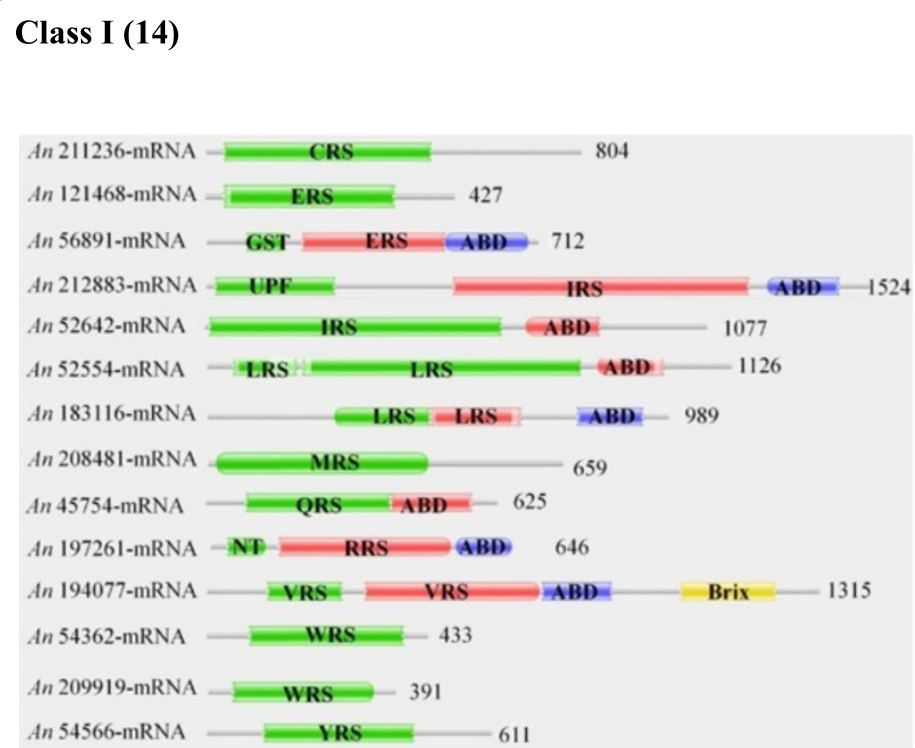

\section{aaRS associated domain}

$$
\text { An 210303-mRNA } \quad \text { GST } 445
$$

Figure 2 Domain annotations for predicted aminoacyl-tRNA synthetases (aaRSs) in A. niger. Catalytic domains are labeled with three letter code for corresponding aaRS and number at the end of sequence denotes the length of the protein. ABD - Anti-codon binding, FDX - Ferridoxin-fold ABD, UPF - Unknown protein family, GST - Glutathione-S-transferase, NT - N-terminal, CT - C-terminal, DUF2156 - Domain of unknown function, SAD - Second associated domain.

observations raise an intriguing possibility for cytoplasmic ValRS (194077-mRNA) to participate in rRNA production. The Brix domain in ValRS (194077-mRNA) appears to be unique to $A$. niger and is not observed in Human ValRSs (Figure $3 \mathrm{~B}$ ). In order to manually annotate the gene for $A$. niger VRS (having Brix domain fusion), BLAST searches for 194077-mRNA were performed against the $A$. niger transcriptome database located at the Broad Institute, USA. A highly significant hit was obtained spanning the fungal full-length protein sequence (Additional file 1: Figure S2A). These results indicate that chromosome 16 within $A$. niger genome codes for VRS-Brix domain fusion protein. Structural modeling for the Brix domain from 194077-mRNA confirms conservation in the catalytic fold with respect to its homologs (Additional file 1: Figure S2B).
The class II set of aaRSs in $A$. niger showed heterogeneity in the relative organization of catalytic and anti-codon binding (ABD) domains. AlaRS, PheRS, GlyRS, HisRS, ProRS and ThrRS had catalytic domains at the N-termini of anticodon binding domain (Figure 2). While in case of LysRS, SerRS and AsxRS the catalytic domain is present at the Cterminal of the anti-codon binding domain (Figure 2). AspRSs (56196-mRNA and 57039-mRNA) were the only class II proteins in $A$. niger for which anti-codon binding domain could not be annotated. However, based on Pfam assignment of catalytic domain in these proteins it appears likely that the ABD lies at the N-terminal region (Figure 2). In case of PheRS, two proteins were found with different predicted sub-cellular localization. Cytoplasmic PheRS was predicted to be a heterodimer comprising of $\alpha$ (56159mRNA) and $\beta$ (211951-mRNA) subunits. On the other 


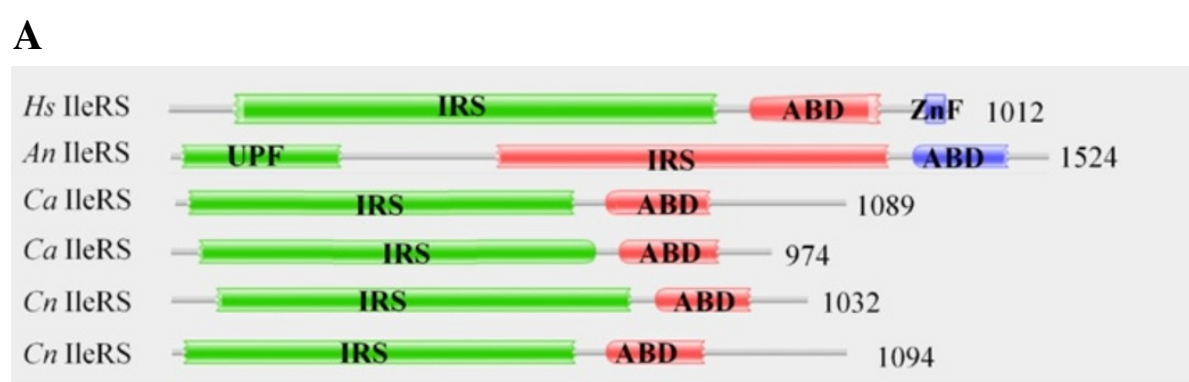

\section{B}

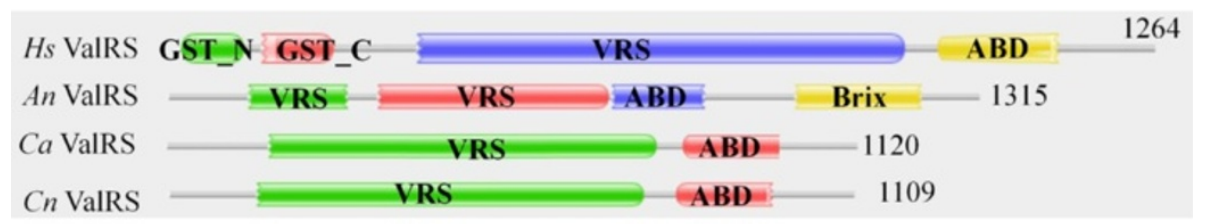

C

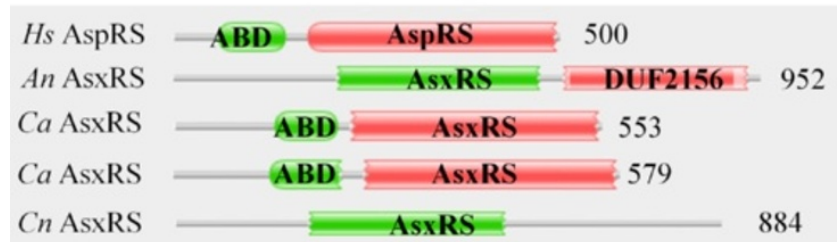

Figure 3 Comparison of domain architectures of $A$. niger and H. sapiens aaRSs. Homologous proteins from C. albicans and C. neoformans are also shown. (A) An uncharacterized protein family (UPF) domain at the N-terminal of A. niger lleRS (212883-mRNA) is absent in the homolog from H. sapiens. A. niger and H. sapiens lleRSs share $\sim 36 \%$ sequence identity. (B) Comparison of domain architectures of ValRS from A. niger (194077-mRNA) and H. sapiens. Brix domain at the C-terminal is unique to A. niger ValRS, and these two ValRSs are $46 \%$ identical. (C) A. niger AsxRS has a unique domain of unknown function (DUF) C-terminal to the catalytic domain. A. niger AsxRS and H. sapiens AspRS are $45 \%$ identical.

hand, mitochondrial PheRS (55517-mRNA) retain a ferrodoxin fold containing anti-codon binding domain (Figure 2). These observations suggest that the quaternary (tetrameric) structure of mitochondrial PherRS (55517-mRNA) comprises only of one protomer while cytoplasmic PheRS has $\alpha \beta$ heterodimer as the basic subunit of the tetramer.

Interestingly, we identified two non-discriminating aspartyl-tRNA synthetases (AsxRS) that were predicted to localize in cytoplasm (207470-mRNA) and nucleus (55404-mRNA) respectively. These enzymes catalyze the charging of both tRNA ${ }^{\text {Asp }}$ and tRNA ${ }^{\text {Asn }}$ with aspartate [51]. Asp-tRNA ${ }^{\text {Asn }}$ thus generated is further acted upon by amidotransferase leading to synthesis of Asn-tRNA ${ }^{\text {Asn }}$ [59]. Curiously, one AsxRS (55404-mRNA), predicted to have nuclear location, has an additional domain of unknown function (DUF) at the C-terminal (Figure 2) which is missing from the human homolog (Figure 3C). Profile HMM searches for the amidotransferases identified two Asn synthase - 57091-mRNA (mitochondrial) and 186429-mRNA (cytoplasmic) (Figure 2). These amidotranferases together with non-discriminating aspartyltRNA synthetase (AsxRS) likely participate in the indirect synthesis of Asn-tRNA ${ }^{\text {Asn }}$. Our results show that there is only one AsnRS which is localized in mitochondria.
Therefore it appears that the mitochondrial repertoire of Asn-tRNA $^{\text {Asn }}$ is maintained by AsnRS as well as by the combined activity of AsxRS and Asn synthase. The cytoplasmic pool of Asn-tRNA ${ }^{\text {Asn }}$ is likely maintained only by the collective activity of AsxRS and Asn synthase enzymes. Homologs for Gln synthase subunits A (170115-mRNA), B (172989-mRNA) and F (173432-mRNA) were also identified (Table 1). These three subunits together constitute an active GatFAB enzyme within the mitochondria and facilitate the synthesis of Gln-tRNA ${ }^{\text {Gln }}$ within the organelle. Curiously, GatF (173432-mRNA) has an additional domain (DUF726) at its C-terminal (Figure 2). It is possible that the mitochondrial GluRS, like its human homolog, is essentially a non-discriminating enzyme that synthesizes Glu-tRNA ${ }^{\text {Gln }}$ which acts as a substrate for heterodimeric Gln synthase to yield Gln-tRNA ${ }^{\text {Gln }}$.

Significant matches for standalone trans-editing and deacylase domains were also observed for two proteins 189390-mRNA and 56197-mRNA that contains AlaX and DTD domains respectively. We also identified a P43 homo$\log$ (210303-mRNA) in A. niger. Interestingly, A. niger $\mathrm{P} 43$ has GST C-terminal domain at the N-terminal of the tRNA binding region, which is different from the human counterpart. Indeed, GST_C domain is absent in 
human P43, however, it is present in P43 homolog from Toxoplasma gondii [17] (Figure 4A). Multiple sequence alignment for P43 homologs shows high conservation in the tRNA binding domains between human, the three fungi, and T. gondii P43s (Figure 4B). However, $A$. niger $\mathrm{P} 43$ sequence has two insertions (one of 4 residues and another of 9 residues) within the structurally conserved tRNA binding domain. Further, the N-termini of these proteins show poor homology amongst each other. Finally, GluRS (56891-mRNA), HisRS (51854-mRNA), and LeuRS (52554-mRNA) were observed to have 'ELR' motifs in their $\mathrm{N}$-terminal regions (Additional file 1: Table S2). In human and other TyrRSs this motif can impart cytokine-related activities [21,60-62] which are untested in the three fungi so far. Mapping of ELR motifs onto the modeled structures showed that the ELR motif appeared in surface exposed loop regions in these three aaRSs (data not shown).

\section{Domain architecture for aaRSs in C. albicans}

The total number of aaRSs identified in C. albicans was 28 with 15 and 13 members in class I and class II respectively. The general organization of domains amongst class I aaRSs was prototypical: catalytic domain followed by anticodon binding domain (Figure 5 ). For $\sim 50 \%$ of class I aaRSs, two different proteins were identified specific for a particular amino acid - presumably one aaRS each for cytoplasm and mitochondria (Figure 5). Class I aaRSs for which only one copy was identified includes CysRS (orf19.4931), MetRS (orf19.3955), GlnRS (orf19.7064), ArgRS (orf19.3341), and ValRS (orf19.1295). The cytoplasmic copy of GluRS (orf19.7057) had an additional glutathione-S-transferase (GST_C) domain appended at the N-terminus (Figure 5), like the human bifunctional glutamate/proline-tRNA synthetase. In case of MetRS (orf19.3955), an additional Nterminal domain was identified based on Pfam annotation. This domain is again unique to C. albicans MetRS and is absent from the other two fungal MetRSs analyzed here as well as from the human MetRS (Figure 5). Two proteins each for IleRS (orf19.2138 and orf19.2382) and LeuRS (orf19.2560 and 5705) were predicted to have anti-codon binding domain at the C-terminal of catalytic domain (Figure 5). Two differentially localized proteins each for TrpRS (orf19.4299 and orf19.5226) and TyrRS (orf19.109 and orf19.2694) were also identified (Table 2 and Figure 5).

Class II aaRSs were generally present as a single protein except for PheRS and AsxRS. The two PheRSs had different predicted sub-cellular localization with one likely in cytoplasm and other in mitochondria. Like the $A$. niger mitochondrial PheRS, C. albicans homolog (orf19.2039) also has anti-codon binding domain at the C-terminal of catalytic domain. Further, cytoplasmic PheRS prototypically encompass $\alpha$ (orf19.2960) and $\beta$ (orf19.2573) subunits (Figure 5). The AlaRS (orf19.5746) has second associated domain (SAD) and DHHA1 domains C-terminal to its catalytic domain (Figure 5). These two domains are characteristic of AlaRSs and contribute to the catalytic activity of the enzyme [63]. ProRS (orf19.6701), in addition to catalytic and anti-codon binding domains, had two additional domains one at its termini: N-terminal (editing domain) and a C-terminal domain. ThrRS (orf19.5685) had TGS (ThrRS, GTPase, and SpoT) and SAD (second additional domain) domains present N-terminal to catalytic domain (Figure 5). Two separate non-discriminating aspartyl-tRNA synthetase (AsxRS) were also identified orf19.6702 and orf19.2407 (Figure 5).

Amongst aaRS-associated proteins, two Asn synthases were identified in C. albicans - orf19.3626 (predicted nuclear) and orf19.198 (predicted cytoplasm). These two enzymes could act in tandem with two AsxRSs to generate Asn-tRNA $^{\text {Asn }}$ in an indirect manner. In addition, subunit components of Gln synthase were also identified - GatA (orf19.3956), GatB (orf19.2494) and GatF (orf19.4092) (Table 2 and Figure 5). C. albicans mitochondrial GluRS (orf19.2415) shares $~ 35 \%$ identity with human mitochondrial GluRS (which is non-discriminating), raising the possibility of indirect synthesis of Gln-tRNA ${ }^{\text {Gln }}$ by the action of Gln synthase on Glu-tRNA ${ }^{\text {Gln }}$. Standalone trans-editing domains like AlaX (orf19.5239) and DTD (orf19.297) were also identified (Figure 5). Our results confirm the presence of P43 homolog (orf19.2422) within the C. albicans genome (Figure 5). Three aaRSs - GluRS, HisRS, and LysRS in $C$. albicans have 'ELR' motif at the $\mathrm{N}$-terminal region (Additional file 1: Table S2). The ELR motifs in these three aaRSs were surface exposed in modeled three-dimensional structures (data not shown).

\section{Domain architecture for aaRSs in C. neoformans}

Profile HMM-based searches identified 26 aaRSs with 13 each belonging to class I or II (Figure 6). Amongst class II aaRSs, two aaRSs were observed only for PheRS and GlnRS. The gene structures for other class II aaRSs were mostly similar to those described for A. niger and C. albicans. Two encoded proteins were observed for IleRS (CNBN1610 and CNBA2140) and TyrRS (CNBA1270 and CNBJ0260) (Table 3). Intriguingly, in one of the TyrRSs (CNBA1270), a SAICAR domain (a protein module that synthesizes 4(N-succinylcarboxamide)-5-aminoimidazole ribonucleotide) was identified towards the C-terminal in fusion with the prototypical TyrRS. Predicted sub-cellular localization suggests this unusual fusion TyrRS (CNBA1270) to be cytoplasmic. Other SAICAR domain containing proteins (such as phospho-ribosyl-amino-imidazole-succino-carboxamide synthase) are involved in de novo purine biosynthetic pathway and catalyze the following reaction [64]:

$$
\begin{aligned}
& \text { ATP }+ \text { L-aspartate }+4 \text { - carboxy }-5 \\
& \quad \text {-aminoimidazole ribonucleotide }(\text { CAIR }) \\
& \rightleftharpoons \mathrm{ADP}+\mathrm{Pi}+\mathrm{SAICAR}
\end{aligned}
$$




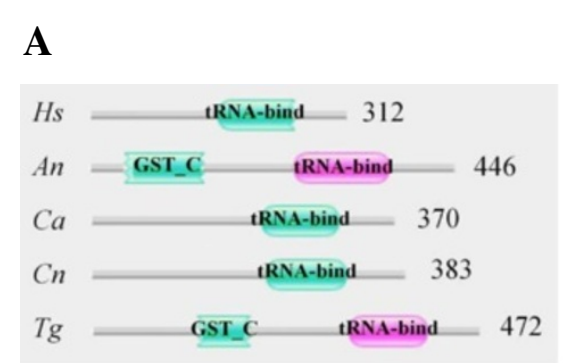

\section{B}

\section{BAD AVG GOOD}

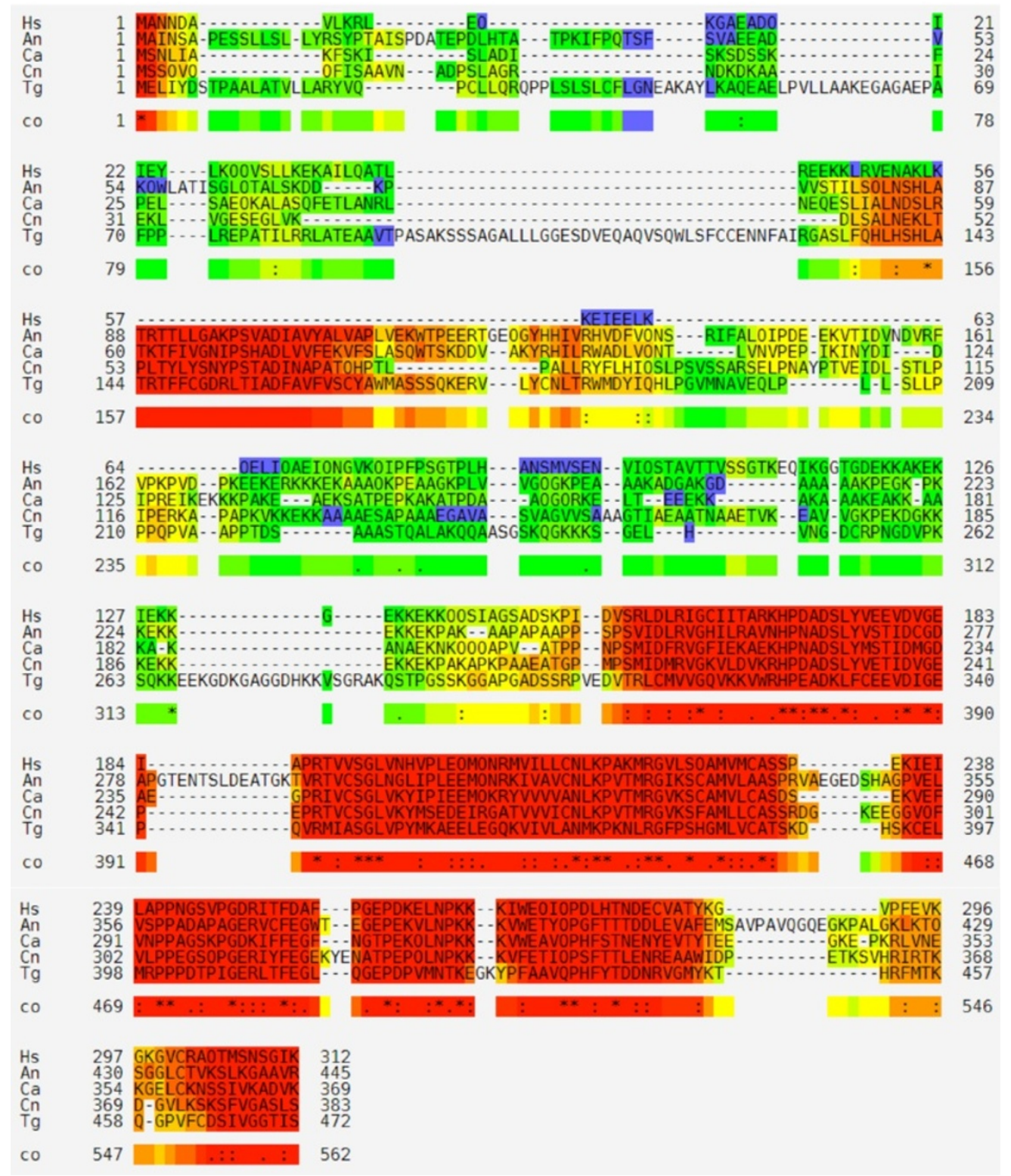

Figure 4 (See legend on next page.) 
(See figure on previous page.)

Figure 4 Comparison of P43 homologs from different genomes. (A) Pfam-based domain organization for P43 homologs from Homo sapiens $(\mathrm{Hs})$, A. niger (An), C. albicans (Ca), C. neoformans (Cn), and Toxoplasma gondii (Tg). (B) Sequence alignment for P43s with color codes showing sequence conservation - low (blue) to high (red). Overall, there is high conservation in the tRNA-binding domain at the C-terminal and poor homology at the $\mathrm{N}$-terminals.

Vertebrate SAICAR synthetases are significantly different from their microbial homologs both in terms of subunit structure (vertebrate SAICAR synthetase are multimeric proteins) and functions (vertebrates have bifunctional enzymes having AIR carboxylase and SAICAR synthetase activity [65]). A recent study has suggested microbial SAICAR synthetases as potential drug target [66]. Significantly, we could not identify a homolog for TyrRS-SAICAR fusion protein (CNBA1270) in humans and hence these unusual fused domains seems unique to C. neoformans (Figure 7A). Intriguingly, BLAST searches against non-redundant databases revealed that homologs for TyrRS-SAICAR fusion protein exist only in some other fungi such as Cryptococcus gatti, Trichosporon asahii and Tremella mesenterica. In order to validate this domain fusion in the case of $C$. neoformans, BLAST searches for CNBA1270 were done against transcriptome database available at BROAD institute, USA. A highly significant transcript was obtained corresponding to the computationally predicted protein sequence (Additional file 1: Figure S3). These results reinforce presence of a chromosomal region in C. neoformans genome that encodes for YRS-SAICAR fusion protein. Modeling of the tertiary structure for SAICAR domain in CNBA1270 confirmed conservation of key residues within the ATP binding pocket (Figure 7B). These observations further substantiate the hypothesis that this fusion domain protein

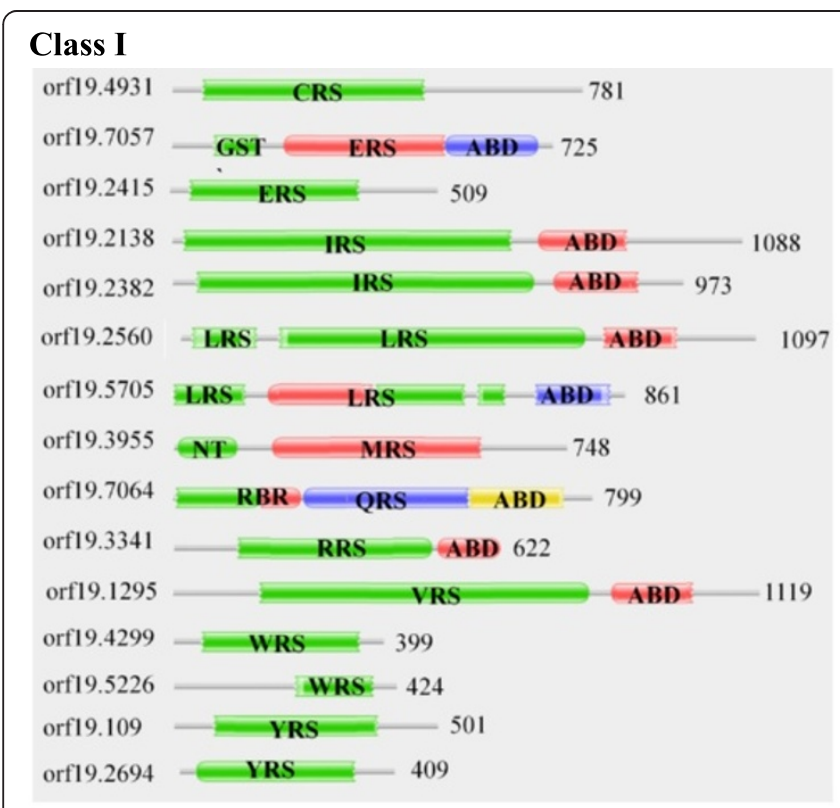

\section{Trans editing}

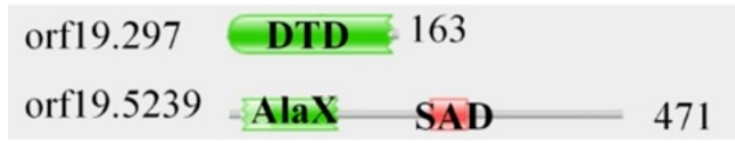

aaRS associated domain

$$
\text { orf19.2422 } \quad \text { tRNA-bind } 369
$$

\section{Class II}

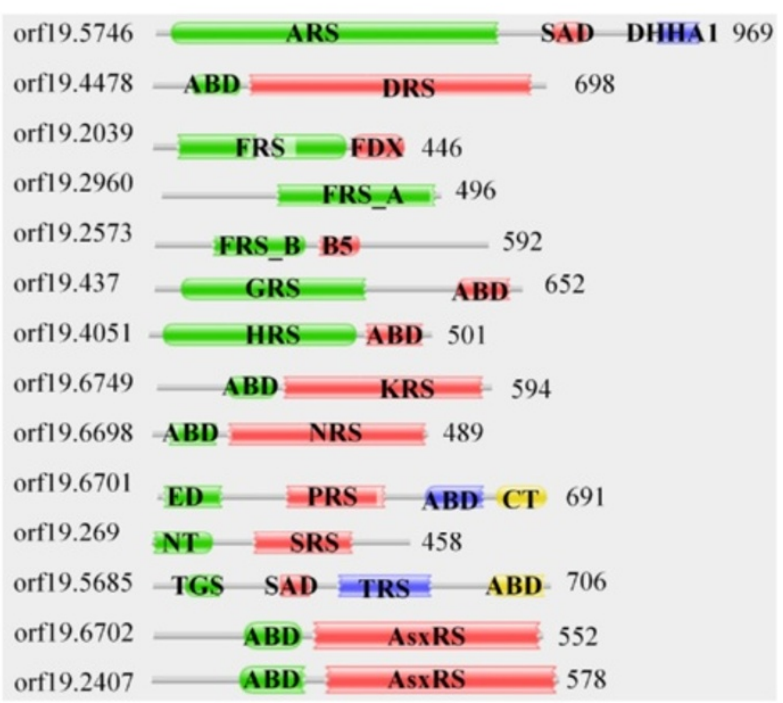

Amidotransferases

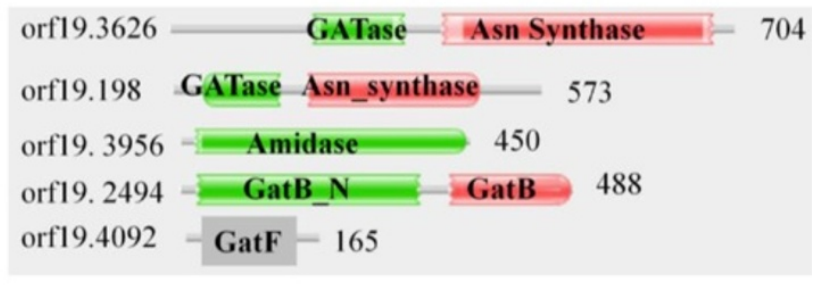

Figure 5 Domain annotations for predicted aminoacyl-tRNA synthetases (aaRSs) in C. albicans. Catalytic domains are labeled with three letter code for corresponding aaRS and number at the end of the sequence denotes the length of the protein. ED - Editing domain, TGS - (ThrRS, GTPase, and SPOT) domain, RBR - non-specific RNA binding region, DHHA1 - DHH associated domain, and other domains are same as in Figure 2. 


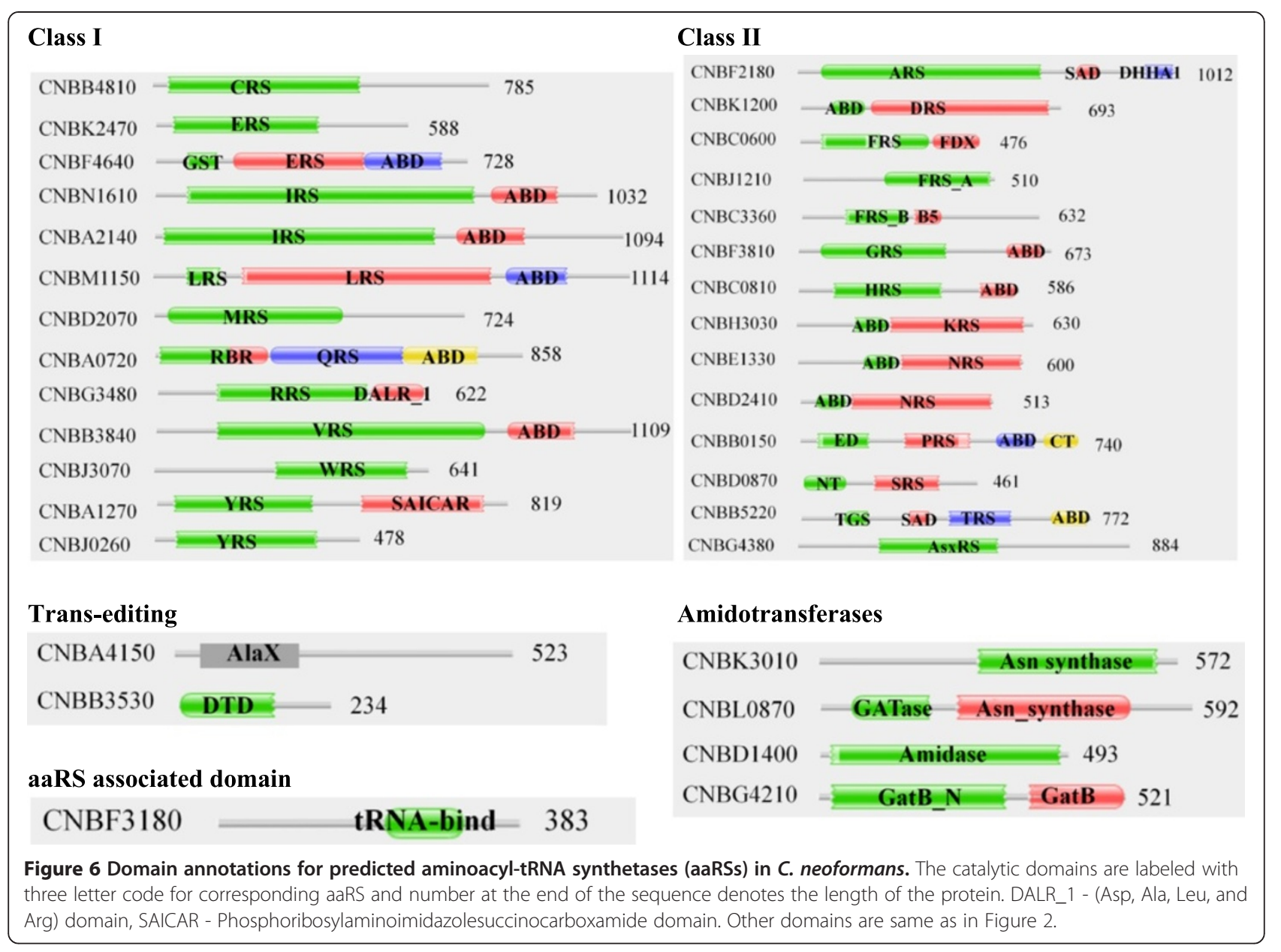

could be potentially targeted for therapeutic development against $C$. neoformans.

Among aaRS related proteins, four polypeptides were predicted to have amidotransferases activity - two Asn synthases (CNBK3010 and CNBL0870) and other two Gln synthases (subunit A (CNBD1400) and subunit B (CNBG4210)). We were not able to identify any homolog for GatF subunit. Interestingly, all these four amidotranferases were predicted to localize within the mitochondria (Table 3). In addition, a standalone trans-editing AlaX (CNBA4150) and DTD (CNBB3530) were also identified (Figure 6). Interestingly, the mitochondrial TyrRS-SAICAR fusion protein (CNBJ0260) had an 'ELR' motif at its Nterminal (Additional file 1: Table S2). This motif in human TyrRS is responsible for cytokine activity. Structural modeling for C. neoformans TyrRS part of the fusion protein suggests that the 'ELR' motif is likely to be solvent accessible (Figure 8A). Further, the 'ELR' motifs in human and C. neoformans TyrRSs are part of $\alpha$ helices that superimpose well (Figure 8B). Presence of ELR motif at the $\mathrm{N}$-terminal and an additional unique SAICAR domain towards C-terminal makes C. neoformans mitochondrial
TyrRS (CNBJ0260) an interesting target for further investigation. In addition, we noted that ProRS (CNBB0150) also has an 'ELR' motif towards its N-terminal (Additional file 1: Table S2), which when modeled indicated surface exposure for this motif.

\section{Conclusions}

Treatment and prevention of opportunistic fungal infections in humans presents an emerging challenge. Aspergillus, Candida and Cryptococcus are three highly pathogenic fungi, particularly in immuno-compromised patients. Dissecting and understanding critical fungal protein machineries is therefore vital for establishing a base for launching new therapeutics against these pathogens. Within microbial proteomes, aminoacyl-tRNA synthetases have already been exploited for drug discovery [67]. Specifically for fungal aaRS, a novel boron-containing molecule 5-fluoro1,3-dihydro-1-hydroxy-2,1-benzoxaborole (AN2690) that inhibits yeast cytoplasmic leucyl-tRNA synthetase by trapping enzyme-bound tRNA ${ }^{\text {Leu }}$ in editing conformation is now an anti-fungal agent in the market [22]. Hence, proof-of-principle exists that can now be expanded to 


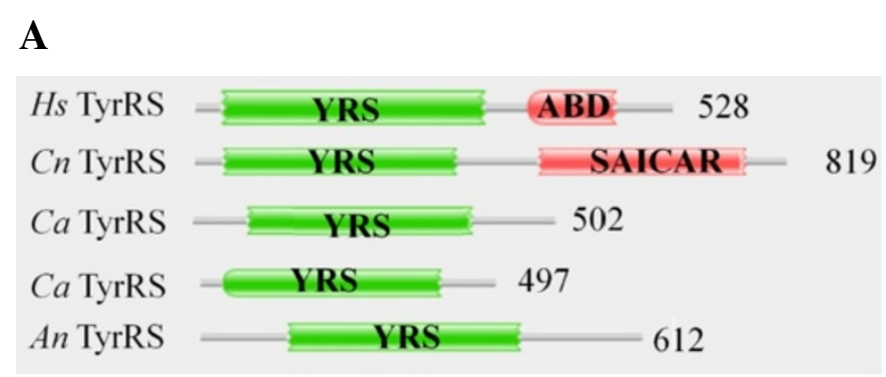

\section{B}

Modelled structure for SAICAR domain in C. neoformans TyrRS

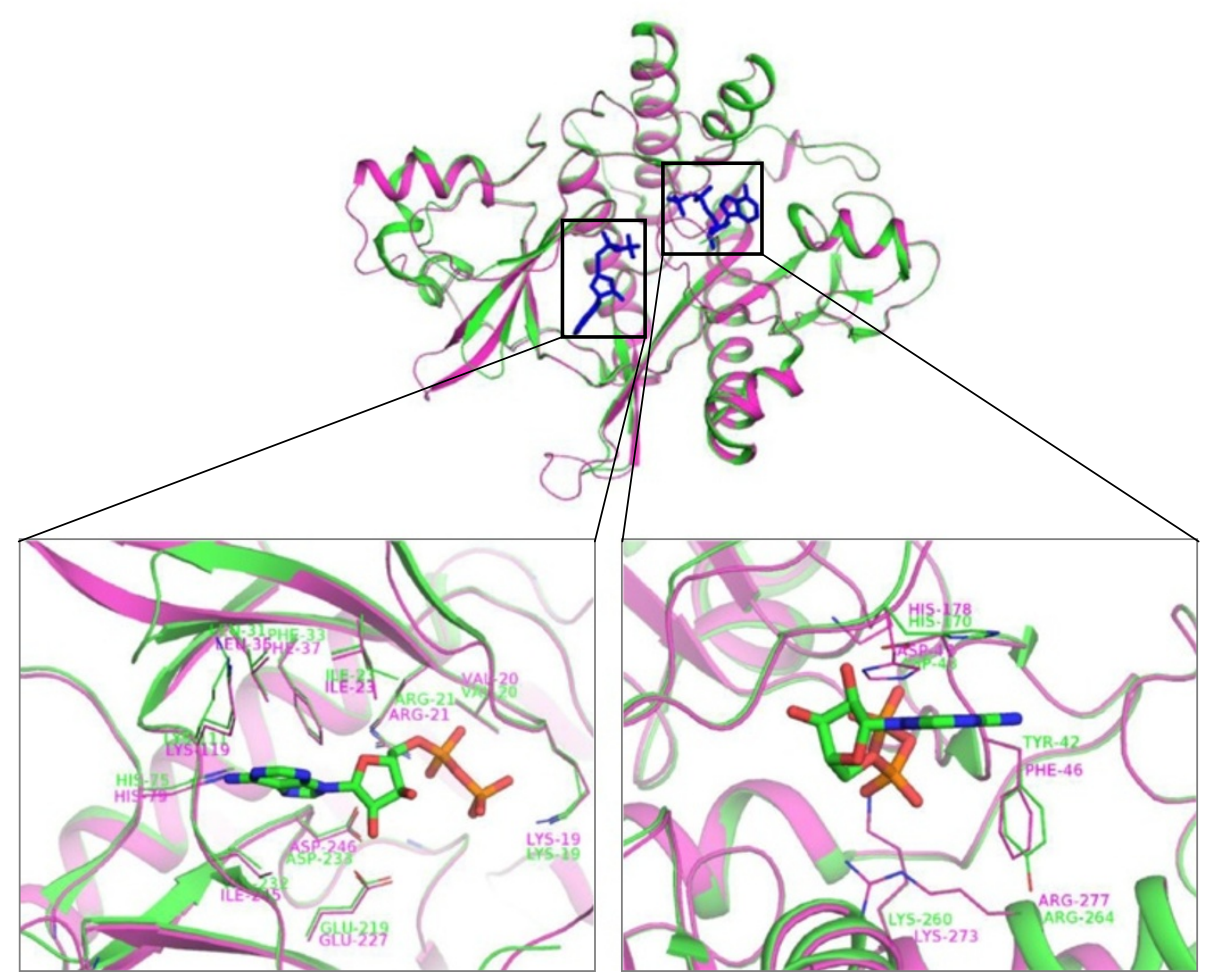

Figure 7 Sequence and structural analyses for tyrosyl-tRNA synthetase from C. neoformans. (A) Comparison of domain architectures for C. neoformans TyrRS (CNBA1270) and H. sapiens cytoplasmic TyrRS based on the Pfam domain assignment. SAICAR domain at the C-terminal of C. neoformans TyrRS is absent in the homolog from H. sapiens and these two TyrRSs are $\sim 47 \%$ identical in the TyrRS catalytic domain region. (B) Superimposition of modeled C. neoformans SAICAR domain in TyrRS (magenta) and crystal structure of SAICAR synthase (PDB ID 2CNQ) from Saccharomyces cerevisiae (green) with adenosine-di-phosphate (ADP, blue). The two proteins share $51 \%$ sequence identity. Inset shows sequence and structural conservation of ADP interacting residue in the two proteins.

target a much larger number of fungal aaRSs. Our profile HMM-based identification and annotation confirms the existence of all 20 aaRSs in the three studied fungal genomes. In addition, fungal DTDs, AlaXs, P43s and amidotransferases have also been comprehensively annotated. Putative distribution of fungal aaRSs in different subcellular compartments within the fungi provides a base for experimental validation. Our results also highlight fungal processes that enable multiple localization of aaRSs and/or of charged tRNAs between cytoplasm and mitochondria in these three fungi since neither their cytoplasm nor mitochondria individually harbor a complete set of 20 aaRSs.

Overall, the three fungal genomes harbor similar number of aaRSs. In A. niger, C. albicans, and C. neoformans there exist 8,7 , and 5 aaRSs that are encoded by two different set of genes, presumably for mitochondrial localization (Figure 1A). PheRS, GluRS, and IleRS are the three aaRSs that are two-gene sets in all the three fungal genomes. The distribution for aaRS associated proteins is conserved 


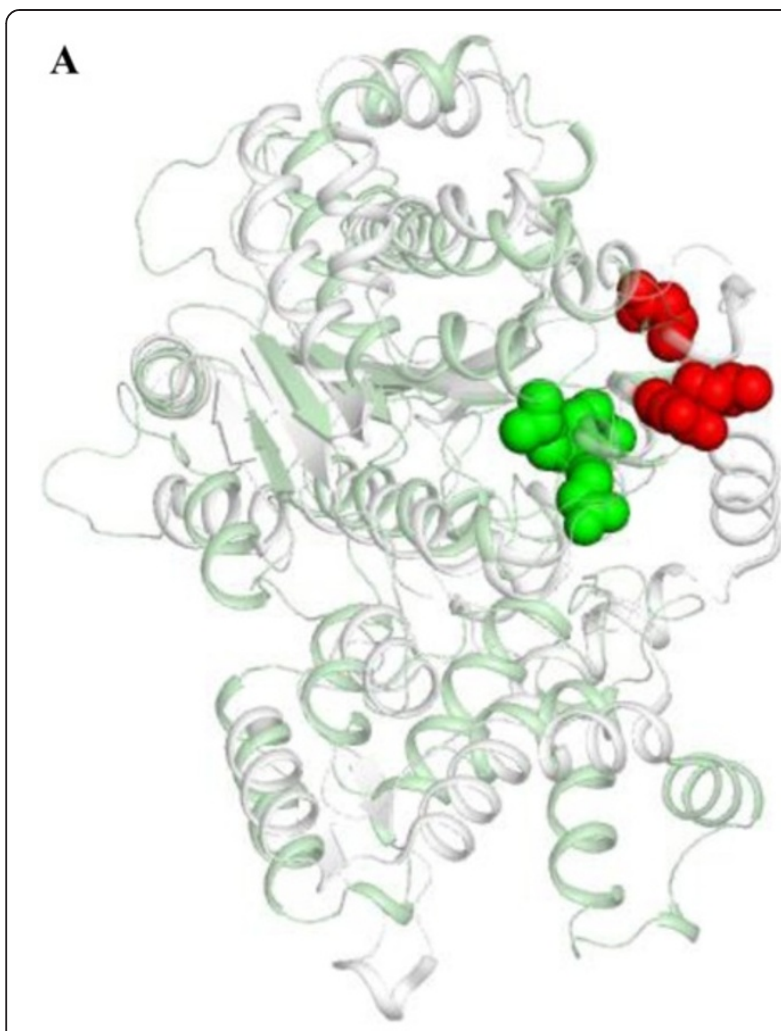

B

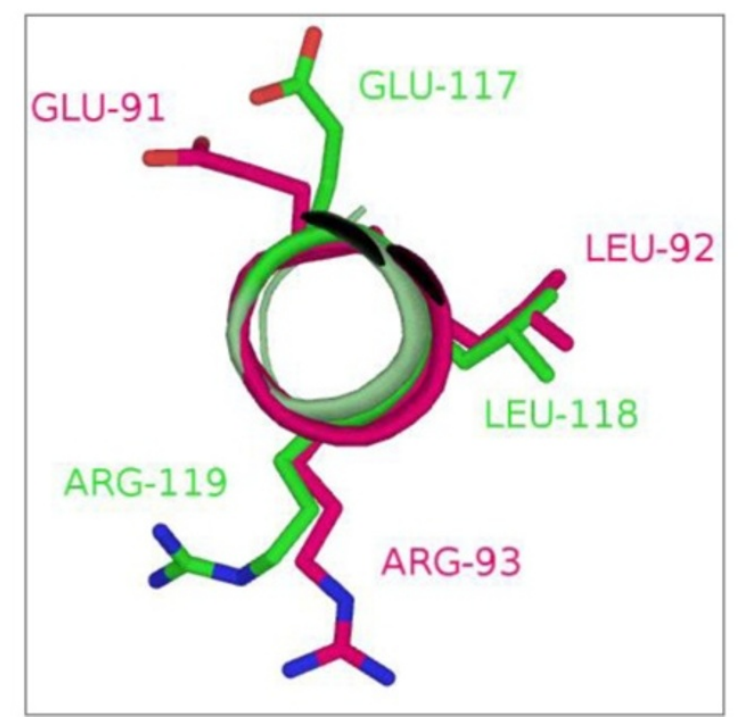

Figure 8 Superimposition for crystal structures of human TyrRS (white, PDBID 1N3L) and modeled structure of C. neoformans TyrRS (light green). (A) Residues constituting ELR motif in the two proteins are shown as spheres in red and green for human and $C$. neoformans, respectively. (B) Superimposition of the ELR motifs from the two proteins.

among the three studies fungi. Our results predict that $C$. neoformans Gln synthase (Gat) maybe heterodimeric unlike its homologs in other two fungi where it is likely to be heterotrimeric. None of the aaRS in C. neoformans were predicted to localize within nucleus while 2 and 6 aaRSs in A. niger and C. albicans were predicted to have nuclear localization (Figure 1B).

The aaRSs identified in these fungal genomes share some similarities with their human homologs (Tables 1, 2 and 3) but also many critical differences (Figures 3 and 6). $A$. niger IleRS and AsxRS have unique domain fusions which are absent in homologs from the other two fungi and from humans (Figure 3). ValRS and TyrRS from $A$. niger and $C$. neoformans also harbor novel functionallycharacterized domain fusions which are absent from humans (Figures 3 and 7). Further structural and functional characterization of the unique and novel fungal aaRSs is required before they can be exploited for development of anti-fungal compounds. In addition, experimental analyses of fungal aaRSs that display unusual domain fusions may uncover their non-canonical functions. We found N-terminal GST_C domains in A. niger P43 which is distinct feature for this protein in comparison to other two fungi and humans (Figure 4). Our results also reveal presence of novel and unique fusion proteins where domains such as DUF2156 and SAICAR are appended to the aaRS structural cores (Figures 2 and 6).

In summary, the comprehensive genomic cataloging of aaRSs from pathogenic fungi detailed here warrants further experimental validation and exploration. The results presented here provide insights into protein translation enzymes within these pathogenic fungi that can be targeted for developing new drugs against these microbes.

\section{Availability of supporting data}

The data sets supporting the results of this article are included within the article and Additional file 1.

\section{Additional file}

Additional file 1: Table S1 and Table S2. Having the details of the dataset used for analyses and summary of fungal aaRSs with ELR motif, respectively. Figure $\mathbf{S} 1$. Shows details for signal sequences identified in fungal aaRSs. Figure S2. Shows (A) schematic representation for genome and transcriptome sequencing analyses for VRS-Brix and (B) modelling for Brix domain from C. neoformans. Figure S3. Schematic representation for genome and transcriptome sequencing analyses for YRS-SAICAR fusion protein in C. neoformans.

\section{Competing interests}

The authors declare that they have no competing interests.

Authors' contributions

AS conceived the study. MD did the experiments, AS and MD analyzed the data. MD wrote the manuscript and AS revised it. Both authors read and approved the final manuscript.

\section{Acknowledgement}

OSRP Department of Biotechnology, India grant to AS supported this work. AS is a JC Bose fellow. 
Received: 18 June 2014 Accepted: 20 November 2014

Published: 5 December 2014

\section{References}

1. Brown GD, Denning DW, Gow NA, Levitz SM, Netea MG, White TC: Hidden killers: human fungal infections. Sci Transl Med 2012, 4(165):165rv113.

2. Brown GD, Denning DW, Levitz SM: Tackling human fungal infections. Science 2012, 336(6082):647.

3. Pfaller MA, Diekema DJ: Rare and emerging opportunistic fungal pathogens: concern for resistance beyond Candida albicans and Aspergillus fumigatus. J Clin Microbiol 2004, 42(10):4419-4431.

4. van Burik JA, Magee PT: Aspects of fungal pathogenesis in humans. Annu Rev Microbiol 2001, 55:743-772.

5. Xie JL, Polvi EJ, Shekhar-Guturja T, Cowen LE: Elucidating drug resistance in human fungal pathogens. Future Microbiol 2014, 9:523-542.

6. Kett DH, Azoulay E, Echeverria PM, Vincent JL: Candida bloodstream infections in intensive care units: analysis of the extended prevalence of infection in intensive care unit study. Crit Care Med 2011, 39(4):665-670.

7. Manfredi R, Calza L, Chiodo F: Dual Candida albicans and Cryptococcus neoformans fungaemia in an AIDS presenter: a unique disease association in the highly active antiretroviral therapy (HAART) era. J Med Microbiol 2002, 51(12):1135-1137.

8. Rinaldi MG: Invasive aspergillosis. Rev Infect Dis 1983, 5(6):1061-1077.

9. Person AK, Chudgar SM, Norton BL, Tong BC, Stout JE: Aspergillus niger: an unusual cause of invasive pulmonary aspergillosis. $J$ Med Microbiol 2010, 59(Pt 7):834-838

10. Chayakulkeeree M, Perfect JR: Cryptococcosis. Infect Dis Clin North Am 2006, 20(3):507-544. v-vi.

11. Botts MR, Giles SS, Gates MA, Kozel TR, Hull CM: Isolation and characterization of Cryptococcus neoformans spores reveal a critical role for capsule biosynthesis genes in spore biogenesis. Eukaryot Cell 2009, 8(4):595-605.

12. Shelburne SA 3rd, Darcourt J, White AC Jr, Greenberg SB, Hamill RJ, Atmar RL, Visnegarwala F: The role of immune reconstitution inflammatory syndrome in AIDS-related Cryptococcus neoformans disease in the era of highly active antiretroviral therapy. Clin Infect Dis 2005, 40(7):1049-1052.

13. Bruno VM, Wang Z, Marjani SL, Euskirchen GM, Martin J, Sherlock G, Snyder M: Comprehensive annotation of the transcriptome of the human fungal pathogen Candida albicans using RNA-seq. Genome Res 2010, 20(10):1451-1458.

14. de Souza WR, de Gouvea PF, Savoldi M, Malavazi I, de Souza Bernardes LA, Goldman MH, de Vries RP, de Castro Oliveira JV, Goldman GH: Transcriptome analysis of Aspergillus niger grown on sugarcane bagasse. Biotechnol Biofuels 2011, 4:40.

15. Janbon G, Ormerod KL, Paulet D, Byrnes EJ 3rd, Yadav V, Chatterjee G, Mullapudi N, Hon CC, Billmyre RB, Brunel F, Bahn YS, Chen W, Chen Y, Chow EW, Coppee JY, Floyd-Averette A, Gaillardin C, Gerik KJ, Goldberg J, Gonzalez-Hilarion S, Gujja S, Hamlin JL, Hsueh YP, Ianiri G, Jones S, Kodira CD, Kozubowski L, Lam W, Marra M, Mesner LD, et al: Analysis of the genome and transcriptome of Cryptococcus neoformans var. grubii reveals complex RNA expression and microevolution leading to virulence attenuation. PLoS Genet 2014, 10(4):e1004261.

16. Ibba M, Soll D: Aminoacyl-tRNA synthesis. Annu Rev Biochem 2000, 69:617-650

17. Ling J, Reynolds N, Ibba M: Aminoacyl-tRNA synthesis and translational quality control. Annu Rev Microbiol 2009, 63:61-78.

18. Perona JJ, Hadd A: Structural diversity and protein engineering of the aminoacyl-tRNA synthetases. Biochemistry 2012, 51(44):8705-8729.

19. Bhatt TK, Kapil C, Khan S, Jairajpuri MA, Sharma V, Santoni D, Silvestrini F, Pizzi E, Sharma A: A genomic glimpse of aminoacyl-tRNA synthetases in malaria parasite Plasmodium falciparum. BMC Genomics 2009, 10:644.

20. Bhatt TK, Khan S, Dwivedi VP, Banday MM, Sharma A, Chandele A, Camacho N, de Pouplana LR, Wu Y, Craig AG, Mikkonen AT, Maier AG, Yogavel M, Sharma A: Malaria parasite tyrosyl-tRNA synthetase secretion triggers pro-inflammatory responses. Nat Commun 2011, 2:530.

21. van Rooyen JM, Murat JB, Hammoudi PM, Kieffer-Jaquinod S, Coute Y, Sharma A, Pelloux H, Belrhali H, Hakimi MA: Assembly of the novel five-component apicomplexan multi-aminoacyl-tRNA synthetase complex is driven by the hybrid scaffold protein Tg-p43. PLoS One 2014, 9(2):e89487.

22. Rock FL, Mao W, Yaremchuk A, Tukalo M, Crepin T, Zhou H, Zhang YK, Hernandez V, Akama T, Baker SJ, Plattner JJ, Shapiro L, Martinis SA,
Benkovic SJ, Cusack S, Alley MR: An antifungal agent inhibits an aminoacyl-tRNA synthetase by trapping tRNA in the editing site. Science 2007, 316(5832):1759-1761.

23. Bhatt TK, Yogavel M, Wydau S, Berwal R, Sharma A: Ligand-bound structures provide atomic snapshots for the catalytic mechanism of D-amino acid deacylase. J Biol Chem 2010, 285(8):5917-5930.

24. Bour T, Akaddar A, Lorber B, Blais S, Balg C, Candolfi E, Frugier M: Plasmodial aspartyl-tRNA synthetases and peculiarities in Plasmodium falciparum. J Biol Chem 2009, 284(28):18893-18903.

25. Hoen R, Novoa EM, Lopez A, Camacho N, Cubells L, Vieira P, Santos M, Marin-Garcia P, Bautista JM, Cortes A, Ribas de Pouplana L, Royo M: Selective inhibition of an apicoplastic aminoacyl-tRNA synthetase from Plasmodium falciparum. Chembiochem 2013, 14(4):499-509.

26. Hoepfner D, McNamara CW, Lim CS, Studer C, Riedl R, Aust T, McCormack SL, Plouffe DM, Meister S, Schuierer S, Plikat U, Hartmann N, Staedtler F, Cotesta S, Schmitt EK, Petersen F, Supek F, Glynne RJ, Tallarico JA, Porter JA, Fishman MC, Bodenreider C, Diagana TT, Movva NR, Winzeler EA: Selective and specific inhibition of the plasmodium falciparum lysyl-tRNA synthetase by the fungal secondary metabolite cladosporin. Cell Host Microbe 2012, 11(6):654-663.

27. Istvan ES, Dharia NV, Bopp SE, Gluzman I, Winzeler EA, Goldberg DE: Validation of isoleucine utilization targets in Plasmodium falciparum. Proc Natl Acad Sci U S A 2011, 108(4):1627-1632.

28. Jackson KE, Pham JS, Kwek M, De Silva NS, Allen SM, Goodman CD, McFadden Gl, de Pouplana LR, Ralph SA: Dual targeting of aminoacyl-tRNA synthetases to the apicoplast and cytosol in Plasmodium falciparum. Int J Parasitol 2012, 42(2):177-186.

29. Keller TL, Zocco D, Sundrud MS, Hendrick M, Edenius M, Yum J, Kim YJ, Lee HK, Cortese JF, Wirth DF, Dignam JD, Rao A, Yeo CY, Mazitschek R, Whitman M: Halofuginone and other febrifugine derivatives inhibit prolyl-tRNA synthetase. Nat Chem Biol 2012, 8(3):311-317.

30. Khan S, Garg A, Camacho N, Van Rooyen J, Kumar Pole A, Belrhali H, Ribas de Pouplana L, Sharma V, Sharma A: Structural analysis of malariaparasite lysyl-tRNA synthetase provides a platform for drug development. Acta Crystallogr D Biol Crystallogr 2013, 69(Pt 5):785-795.

31. Khan S, Sharma A, Jamwal A, Sharma V, Pole AK, Thakur KK: Uneven spread of cis- and trans-editing aminoacyl-tRNA synthetase domains within translational compartments of P. falciparum. Sci Rep 2011, 1:188.

32. Koh CY, Kim JE, Napoli AJ, Verlinde CL, Fan E, Buckner FS, Van Voorhis WC, Hol WG: Crystal structures of Plasmodium falciparum cytosolic tryptophanyl-tRNA synthetase and its potential as a target for structureguided drug design. Mol Biochem Parasitol 2013, 189(1-2):26-32.

33. Mailu BM, Ramasamay G, Mudeppa DG, Li L, Lindner SE, Peterson MJ, DeRocher AE, Kappe SH, Rathod PK, Gardner MJ: A nondiscriminating glutamyl-tRNA synthetase in the plasmodium apicoplast: the first enzyme in an indirect aminoacylation pathway. J Biol Chem 2013, 288(45):32539-32552.

34. Pham JS, Dawson KL, Jackson KE, Lim EE, Pasaje CF, Turner KE, Ralph SA: Aminoacyl-tRNA synthetases as drug targets in eukaryotic parasites. Int J Parasitol Drugs Drug Resist 2014, 4(1):1-13.

35. Pham JS, Sakaguchi R, Yeoh LM, De Silva NS, McFadden GI, Hou YM, Ralph SA: A dual-targeted aminoacyl-tRNA synthetase in Plasmodium falciparum charges cytosolic and apicoplast tRNACys. Biochem J 2014, 458(3):513-523.

36. Gowri VS, Ghosh I, Sharma A, Madhubala R: Unusual domain architecture of aminoacyl tRNA synthetases and their paralogs from Leishmania major. BMC Genomics 2012, 13:621.

37. Rocha R, Pereira PJ, Santos MA, Macedo-Ribeiro S: Unveiling the structural basis for translational ambiguity tolerance in a human fungal pathogen. Proc Natl Acad Sci U S A 2011, 108(34):14091-14096.

38. Stajich JE, Harris T, Brunk BP, Brestelli J, Fischer S, Harb OS, Kissinger JC, Li W, Nayak V, Pinney DF, Stoeckert CJ Jr, Roos DS: FungiDB: an integrated functional genomics database for fungi. Nucleic Acids Res 2012 , 40(Database issue):D675-D681.

39. Consortium U: Activities at the Universal Protein Resource (UniProt). Nucleic Acids Res 2014, 42(Database issue):D191-D198.

40. Hughey R, Krogh A: Hidden Markov models for sequence analysis: extension and analysis of the basic method. Comput Appl Biosci 1996, 12(2):95-107.

41. Punta M, Coggill PC, Eberhardt RY, Mistry J, Tate J, Boursnell C, Pang N, Forslund K, Ceric G, Clements J, Heger A, Holm L, Sonnhammer EL, Eddy SR, Bateman A, Finn RD: The Pfam protein families database. Nucleic Acids Res 2012, 40(Database issue):D290-D301. 
42. Horton P, Park KJ, Obayashi T, Fujita N, Harada H, Adams-Collier CJ, Nakai K: WoLF PSORT: protein localization predictor. Nucleic Acids Res 2007, 35(Web Server issue):W585-W587.

43. Claros MG, Vincens P: Computational method to predict mitochondrially imported proteins and their targeting sequences. Eur J Biochem 1996, 241(3):779-786.

44. Nielsen $\mathrm{H}$, Engelbrecht J, Brunak S, von Heijne G: Identification of prokaryotic and eukaryotic signal peptides and prediction of their cleavage sites. Protein Eng 1997, 10(1):1-6.

45. Notredame C, Higgins DG, Heringa J: T-Coffee: a novel method for fast and accurate multiple sequence alignment. $J \mathrm{Mol}$ Biol 2000, 302(1):205-217.

46. Kelley LA, Sternberg MJ: Protein structure prediction on the Web: a case study using the Phyre server. Nat Protoc 2009, 4(3):363-371.

47. Schrodinger LLC: The PYMOL molecular graphics system, version 1.3r1. 2010.

48. Nabholz CE, Horn EK, Schneider A: tRNAs and proteins are imported into mitochondria of Trypanosoma brucei by two distinct mechanisms. Mol Biol Cell 1999, 10(8):2547-2557.

49. Zheng G, Liu W, Gong Y, Yang H, Yin B, Zhu J, Xie Y, Peng X, Qiang B, Yuan J: Human D-Tyr-tRNA(Tyr) deacylase contributes to the resistance of the cell to D-amino acids. Biochem J 2009, 417(1):85-94

50. Ahel I, Korencic D, Ibba M, Soll D: Trans-editing of mischarged tRNAs. Proc Natl Acad Sci U S A 2003, 100(26):15422-15427.

51. Chuawong $P$, Hendrickson TL: The nondiscriminating aspartyl-tRNA synthetase from Helicobacter pylori: anticodon-binding domain mutations that impact tRNA specificity and heterologous toxicity. Biochemistry 2006, 45(26):8079-8087.

52. Echevarria L, Clemente P, Hernandez-Sierra R, Gallardo ME, Fernandez-Moreno MA, Garesse R: Glutamyl-tRNAGIn amidotransferase is essential for mammalian mitochondrial translation in vivo. Biochem J 2014, 460(1):91-101.

53. Lee SW, Cho BH, Park SG, Kim S: Aminoacyl-tRNA synthetase complexes: beyond translation. J Cell Sci 2004, 117(Pt 17):3725-3734.

54. Wolfe CL, Warrington JA, Davis S, Green S, Norcum MT: Isolation and characterization of human nuclear and cytosolic multisynthetase complexes and the intracellular distribution of p43/EMAPII. Protein Sci 2003, 12(10):2282-2290.

55. Raina M, Elgamal S, Santangelo TJ, Ibba M: Association of a multi-synthetase complex with translating ribosomes in the archaeon Thermococcus kodakarensis. FEBS Lett 2012, 586(16):2232-2238.

56. Guo M, Ignatov M, Musier-Forsyth K, Schimmel P, Yang XL: Crystal structure of tetrameric form of human lysyl-tRNA synthetase: implications for multisynthetase complex formation. Proc Natl Acad Sci U S A 2008 105(7):2331-2336.

57. Park SG, Choi EC, Kim S: Aminoacyl-tRNA synthetase-interacting multifunctional proteins (AIMPs): a triad for cellular homeostasis. IUBMB Life 2010, 62(4):296-302.

58. Eisenhaber F, Wechselberger C, Kreil G: The Brix domain protein family a key to the ribosomal biogenesis pathway? Trends Biochem Sci 2001, 26(6):345-347.

59. Charron C, Roy H, Blaise M, Giege R, Kern D: Non-discriminating and discriminating aspartyl-tRNA synthetases differ in the anticodon-binding domain. EMBO J 2003, 22(7):1632-1643.

60. Ewalt KL, Schimmel P: Activation of angiogenic signaling pathways by two human tRNA synthetases. Biochemistry 2002, 41(45):13344-13349.

61. Wakasugi K, Schimmel P: Two distinct cytokines released from a human aminoacyl-tRNA synthetase. Science 1999, 284(5411):147-151.

62. Wakasugi K, Slike BM, Hood J, Otani A, Ewalt KL, Friedlander M, Cheresh DA, Schimmel P: A human aminoacyl-tRNA synthetase as a regulator of angiogenesis. Proc Natl Acad Sci U S A 2002, 99(1):173-177.

63. Guo M, Chong YE, Beebe K, Shapiro R, Yang XL, Schimmel P: The C-Ala domain brings together editing and aminoacylation functions on one tRNA. Science 2009, 325(5941):744-747.

64. Levdikov VM, Barynin W, Grebenko Al, Melik-Adamyan WR, Lamzin VS, Wilson KS: The structure of SAICAR synthase: an enzyme in the de novo pathway of purine nucleotide biosynthesis. Structure 1998, 6(3):363-376.

65. Ginder ND, Binkowski DJ, Fromm HJ, Honzatko RB: Nucleotide complexes of Escherichia coli phosphoribosylaminoimidazole succinocarboxamide synthetase. J Biol Chem 2006, 281(30):20680-20688.
66. Samant S, Lee H, Ghassemi M, Chen J, Cook JL, Mankin AS, Neyfakh AA: Nucleotide biosynthesis is critical for growth of bacteria in human blood. PLoS Pathog 2008, 4(2):e37.

67. Hurdle JG, O'Neill AJ, Chopra I: Prospects for aminoacyl-tRNA synthetase inhibitors as new antimicrobial agents. Antimicrob Agents Chemother 2005, 49(12):4821-4833.

doi:10.1186/1471-2164-15-1069

Cite this article as: Datt and Sharma: Novel and unique domains in aminoacyl-tRNA synthetases from human fungal pathogens Aspergillus niger, Candida albicans and Cryptococcus neoformans. BMC Genomics 2014 15:1069.

\section{Submit your next manuscript to BioMed Central and take full advantage of:}

- Convenient online submission

- Thorough peer review

- No space constraints or color figure charges

- Immediate publication on acceptance

- Inclusion in PubMed, CAS, Scopus and Google Scholar

- Research which is freely available for redistribution

Submit your manuscript at www.biomedcentral.com/submit
C) BioMed Central 